\title{
Upregulated SPOCK2 Predicts a Worse Overall Survival and Correlates with Abundance of Tumor Infiltrating Immune Cells for High-Grade Serous Ovarian Cancer
}

Jiatao Hao

Xi'an Jiaotong University Medical College First Affiliated Hospital

Yumeng Cao

Baoji Central Hospital

Xiaoya Gu

Xi'an Jiaotong University Medical College First Affiliated Hospital

Minghai Ma

Xi'an Jiaotong University Medical College First Affiliated Hospital

\section{Weihong Wang}

Xi'an Jiaotong University Medical College First Affiliated Hospital

\section{Yan Xue}

Xi'an Jiaotong University Medical College First Affiliated Hospital

Ruifang An ( $\square$ anruifang@xjtu.edu.cn )

Xi'an Jiaotong University Medical College First Affiliated Hospital https://orcid.org/0000-0002-60611706

Research article

Keywords: SPOCK2, high-grade serous ovarian cancer (HGSOC), biomarker, prognosis, extracellular matrix, tumor infiltrating lymphocytes (TILs)

Posted Date: September 30th, 2021

DOI: https://doi.org/10.21203/rs.3.rs-885744/v1

License: (1) This work is licensed under a Creative Commons Attribution 4.0 International License. Read Full License 


\section{Abstract \\ Background}

SPOCK2 is a member of the SPOCK family, a 424-amino acid protein that binds to glycosaminoglycans to form proteoglycans. The purpose of this study was to explore expression profile of SPOCK2, and evaluate prognostic potential and its correlation with immune infiltration in high-grade serous ovarian cancer (HGSOC).

\section{Methods}

Expression of SPOCK2 mRNA and protein between normal and tumor tissues were analyzed using the Cancer Genome Atlas database (TCGA), Gene Expression Omnibus (GEO), Clinical Proteomic Tumor Analysis Consortium (CPTAC), and the Human Protein Atlas (HPA) databases. Receiver operating characteristic (ROC) curve was used to evaluate diagnostic performance of SPOCK2. Kaplan-Meier method and Cox regression analysis were conducted to assess the effect of SPOCK2 on survival. Nomogram was used to predict the impact of SPOCK2 on prognosis. LinkedOmics were used to find correlated genes and perform functional enrichment analyses. The relationships between SPOCK 2 and tumor infiltrating lymphocytes (TILs) were determined by tumor-immune system interaction database (TISIDB) and GSVA package (V1.34.0).

\section{Results}

SPOCK2 was highly expressed in HGSOC tissue compared to normal tissue at both mRNA $(P<0.001)$ and protein $(P=0.03)$ levels. The area under the curve (AUC) is $0.894(\mathrm{Cl}: 0.865-0.923)$. Kaplan-Meier analysis showed that HGSOC patients with high-level SPOCK2 mRNA expression had a worse overall survival (OS) than those with a low expression $(H R=1.45, P=0.005)$. Univariate logistic regression analysis found that age, primary therapy outcome, tumor status, tumor residual, and SPOCK2 expression level were significantly associated with OS $(P<0.05)$. The nomogram model indicated an effective predictive performance of SPOCK2. Kyoto encyclopedia of genes and genomes (KEGG) and gene ontology (GO) term analyses showed that SPOCK2 were mainly involved in regulating extracellular matrix. Immune infiltration analysis showed that SPOCK2 may correlate with abundance of TILs.

\section{Conclusion}

SPOCK2 has potentials to estimate diagnosis and prognosis for HGSOC and is involved in regulating extracellular matrix and immune cell infiltration.

\section{Introduction}


High-grade serous ovarian cancer (HGSOC) is of particular interest, as it comprises over $70 \%$ of epithelial ovarian cancer (EOC) and accounts for the majority of deaths within the first 3 months of ovarian cancer diagnoses, indicating high morbidity and mortality that caused by late-stage presentation and insufficient therapies (Kuroki and Guntupalli, 2020; Lheureux et al, 2019). About 75\% of newly-diagnosed EOC patients are at an advanced stage due to a lack of early and typical warning signs or symptoms as well as effective screening strategies, with 29\% 5-year survival (Kurnit et al. 2021; Reid et al. 2017). Although debulking surgery and platinum/taxane-based chemotherapy regimens are given to delay disease progression or cure the cancer, approximately $70 \%$ of all ovarian cancer patients will succumb to the disease to enter a recurrent or relapse setting (Einzig et al. 1992; Hamanishi et al. 2015; Lheureux et al. 2019). In addition to systemic therapy that maximizes the effect of killing cancer cells in a platinumsensitive setting, poly (ADP-ribose) polymerase inhibitors (PARPi) have presented remarkable benefit in women with recurrent HGSOC regardless of BRCA mutation status, by contrast with improved activity in patients harboring BRCA mutations and platinum-sensitive disease (González-Martín et al. 2019; Mirza et al. 2016; Oza et al. 2018). Nevertheless, multiple acquired resistance mechanisms to PARPi and potential drug toxicities have retarded clinical efficacy, suggesting that efforts might be leveraged into novel researches to delineate resistance mechanisms, prompt drug responses, or find new and specific prognostic biomarkers and therapeutic targets (Hao et al. 2021; Kubalanza and Konecny 2020).

SPARC (osteonectin), cwcv and kazal-like domains proteoglycan 2 (SPOCK2), also known as testican-2, is a member of the SPARC family composing of FSTL1, SMOC1, SMOC2, SPARC, SPARCL1, SPOCK1, SPOCK2, and SPOCK3 (Bradshaw et al. 2012; Said et al. 2008). The protein encoded by SPOCK2 gene contains thyroglobulin type 1 , follistatin-like, and calcium-binding domains as well as glycosaminoglycan binding sites in the acidic $\mathrm{C}$-terminal region, which contribute to form a glycoprotein to orchestrate extracellular matrix and cell membranes (Vannahme et al. 1999). In addition to modulating extracellular matrix formation, SPOCK2 can serve as receptors for growth factors and regulate cellular differentiation and growth (Vannahme et al. 1999). Furthermore, abnormal expression of SPOCK2 have been correlated with tumor growth, invasion, and migration in various human cancers. Previous studies reported that upregulation of SPOCK2 can decrease invasion and metastasis of prostate cancer and endometrial cancer by inhibiting matrix metalloproteinase 2 (MMP2) and membrane-type 1 matrix metalloproteinase (MT1-MMP) (Liu et al. 2019; Ren et al. 2020). Efforts have been made to understand the role of SPOCK2 in tumorigenesis, yet the relationship between SPOCK2 and anti-tumor immunity should also be investigated because extracellular matrix was reported to regulate immune microenvironment surrounding the tumor mass (Crespo et al. 2018; Sorokin et al. 2010). Therefore, studies must be conducted to address the functional contribution of SPOCK2 to tumor immunity, particularly in ovarian cancer.

Given the high expression level of PD-L1 in ovarian cancer, immune checkpoint blockades targeting cytotoxic T-lymphocyte-associated antigen 4 (CTLA-4) or PD-1/PD-L1 have shown somber response rates of approximately $10-15 \%$, indicating repressed activity of immunotherapy (Lheureux et al. 2019; Pujade-Lauraine et al. 2017). In addition to tumor cells and immune effectors, extracellular matrix was found to contribute to drug resistance of immune checkpoint inhibitors (Gordon-Weeks and Yuzhalin 
2020). To incorporate extracellular matrix on cancer immunity and prognosis in HGSOC, we could proceed with SPOCK2. In this study, we first evaluated the patient characteristics and expression of SPOCK2 using the Cancer Genome Atlas (TCGA) and Genotype Tissue Expression (GTEx) datasets, and subsequently examined association between clinical features and prognostic significance of SPOCK2 using Cox regression analysis. Furthermore, we explored the positively and negatively correlated genes of SPOCK2 in HGSOC, and conducted kyoto encyclopedia of genes and genomes (KEGG) pathway analysis and gene ontology (GO) term analysis. Finally, the impact of SPOCK2 on tumor-infiltrating immune cells were analyzed. Our study is important in improving understandings of SPOCK2 in HGSOC and finds that SPOCK2 might be a potential prognostic and therapeutic biomarker for HGSOC.

\section{Material And Methods}

\section{Data acquisition and preprocessing}

RNA-seq transcriptomic data of normal and tumor tissues were respectively downloaded from GTEx and TCGA datasets using UCSC XENA (https://xenabrowser.net/datapages/) to analyze expression level of SPOCK2. The RNA-seq data with workflow type of HTSeq-FPKM (high throughput sequencing-fregments per kilobase per million) were transformed to TPM (transcription per million reads) format which was processed via the Toil procedure (Vivian et al. 2017), and the subsequent log2 conversions were performed for the following analyses. Furthermore, the corresponding patients' clinical information from HGSOC samples were downloaded from TCGA dataset (https://portal.gdc.cancer.gov). Since all gene expression data and patient information were downloaded using R (V3.6.3), the consent from the Ethics and Committee was waived.

\section{Differential expression of SPOCK2}

The ggplot2 package (V3.3.3) was used to visualize differential expression of SPOCK2. RNA sequencing data of 8,295 normal and 9,807 tumor tissues was preprocessed, and the Wilcoxon rank sum test was applied to analyze pan-cancer expression level of SPOCK2 in non-paired samples. The Weltch t' test was subsequently used to assess the differential mRNA expression of SPOCK2 between 88 normal ovary tissues and 427 HGSOC samples. In addition, we performed UALCAN (http://www.ualcan.path.uab.edu/analysis.html) to present differential mRNA expression of SPOCK2 according to clinical features (Chandrashekar et al. 2017). To identify protein profile of SPOCK2 between normal ovary tissues and HGSOC samples, the human protein atlas (HPA) (https://www.proteinatlas.org) was used to download immunohistochemical images. With the application of proteomic technologies, we used the clinical proteomic tumor analysis consortium (CPTAC) (http://www.ualcan.path.uab.edu/analysis-prot.html) to quantify SPOCK2 protein and find associations of SPOCK2 protein level with clinical features (Edwards et al. 2015). The association between SPOCK2 mRNA expression level and clinical characteristics were analyzed with Fisher test or Chi-square test and logistic regression. The diagnostic role of SPOCK2 in HGSOC was assessed by receptor operating characteristic (ROC) curve analysis based on TCGA data. 


\section{Statistical analysis on prognosis of SPOCK2}

The survminer package (V0.4.9) was used to evaluate prognostic potential of SPOCK2 and the cut-off point of SPOCK2 expression was determined by its median value. To investigate the effect of SPOCK2 expression level along with other clinical factors (age, race, FIGO stage, and histologic grade) on OS in TCGA patients, the Kaplan-Meier method with a two-sided log-rank test was used. Furthermore, the survival package (V3.2-10) and the Cox regression analysis was applied to calculate the effect of SPOCK2 expression level and other clinicopathological features (age, race, FIGO stage, histologic stage, primary therapy outcome, tumor status, tumor residual, lymphatic invasion, venous invasion, and anatomic neoplasm subdivision) on survival. The independent prognostic factors derived from multivariate analysis were employed to predict 1-, 3-, and 5-year survival probability, which were visualized using a nomogram. Moreover, the nomogram-predicted probabilities were graphically demonstrated by calibration curves. The concordance index (C-index) was used to assess the discrimination of the nomogram, which was calculated by a bootstrap approach with 1,000 resamples. Notably, nomograms and calibration curves were generated by the rms (V6.2-0) and survival (V3.2-10) packages. All statistical tests were considered as significant if $P$-values were less than 0.05 .

\section{Co-expressed genes and functional enrichment}

LinkedOmics (http://www.linkedomics.org/login.php) is a publicly available dataset that contains multiomics data from all 32 cancer types and a total of 11,158 TCGA patients (Vasaikar et al. 2018). The web application has three analytical modules including LinkFinder, LinkInterpreter, and LinkCompare. Coexpressed genes related to SPOCK2 were identified from the TCGA-OV cohort through the LinkFinder module. The association results were examined by the Pearson correlation coefficient and a Z-score, which were presented in a volcano plot and heat maps, respectively. In the LinkInterpreter module, functional enrichment analyses of KEGG and GO pathways by the gene set enrichment analysis (GSEA) were performed.

\section{Analysis of immune cell infiltration}

We first construct an overall insight of immune cell infiltration related to SPOCK2 using TISIDB (http://www.cis.hku.hk/TISIDB/index.php) which is a web portal for tumor-immune system interactions and integrates multiple heterogeneous data types (Ru et al. 2019). In this study, we performed TISIDB to determine relations between abundance of tumor-infiltrating lymphocytes (TILs) and expression level of SPOCK2 across human cancers. For HGSOC, the relative abundance of 24 immune cell types based on SPOCK2 expression profile was inferred by using SSGSEA that was incorporated in the GSVA package (V1.34.0). To explore the correlation between SPOCK2 mRNA expression level and the enrichment of TILs, scatter plots and Spearman correlation were adopted. Finally, a $t$ test was applied to examine the difference of enrichment score of immune cells between SPOCK2-low and -high groups. All statistical tests were two tailed with a statistical significance level cut-off at 0.05 .

\section{Results}




\section{Pan-cancer analyses of SPOCK2 expression}

We first carried out pan-cancer analyses to evaluate the SPOCK2 mRNA expression profile in the tumor samples of TCGA and the normal samples of GTEx combined with TCGA across different cancer types. The differential expression analyses of SPOCK2 mRNA was finally referred to 33 cancer types by using Wilcoxon rank sum test (Fig. 1). Compared with normal tissues, SPOCK2 was significantly upregulated in breast infiltrating carcinoma (BRCA), cholangiocarcinoma ( $\mathrm{CHOL}$ ), diffuse large $\mathrm{B}$ cell lymphoma (DLBCL), esophageal carcinoma (ESCA), head and neck squamous cell carcinoma (HNSC), acute myeloid leukemia (LAML), brain low grade glioma (LGG), liver hepatocellular carcinoma (LIHC), ovarian serous cystadenocarcinoma (OV), pancreatic cancer (PAAD), pheochromocytoma and paraganglioma (PCPG), skin melanoma (SKCM), gastric cancer (STAD), thyroid cancer (THCA), thymic cancer (THYM), endometrial cancer (UCEC), uterine sarcoma (UCS) $(P<0.05)$; by contrast, SPOCK2 was significantly downregulated in drenocortical carcinoma (ACC), colon adenocarcinoma (COAD), pleomorphic glioma (GBM), renal chromophobe cell carcinoma $(\mathrm{KICH})$, renal clear cell carcinoma (KIRC), renal papillary cell carcinoma (KIRP), lung adenocarcinoma (LUAD), lung squamous cell carcinoma (LUSC), rectum adenocarcinoma (READ), testicular germ cell tumors (TGCT) $(P<0.05)$.

\section{Upregulated expression of SPOCK2 in HGSOC}

To analyze the mRNA and protein expression level of SPOCK2 in HGSOC, we compare the expression data from TCGA and HPA datasets. The non-paired data analysis found that the mRNA expression level of SPOCK2 in 427 TCGA HGSOC samples was significantly higher than those in 88 GTEx normal ovarian samples ( $5.265 \pm 1.691$ vs. $2.795 \pm 1.008, P<0.001$, Fig. $2 A)$. Moreover, a CPTAC analysis was performed to verify the proteomic expression profile of SPOCK2. Compared with 25 normal tissues, 100 ovarian cancer samples were found to have a comparatively higher level of SPOCK2 protein expression $(P=0.03$, Fig. 2B). As shown in Fig. 2D-F, the immunohistochemical staining images from HPA, on the other hand, confirmed that SPOCK2 protein was upregulated in ovarian cancer tissue. In general, these results indicated that both mRNA and protein expression levels of SPOCK2 were upregulated in HGSOC. In addition, the ROC curve was used to determine the distinguishing efficacy of SPOCK2 between normal ovarian tissues $(n=88)$ and HGSOC tissues $(n=427)$ (Fig. 2C). The area under the curve (AUC) of SPOCK2 is 0.894 (Cl: $0.865-0.923)$, suggesting that SPOCK2 is a potential identification marker for HGSOC tissues.

\section{Relationships between SPOCK2 expression and clinical features}

In order to evaluate the relationships between expression level of SPOCK2 and clinical pathological characteristics of ovarian cancer samples, we used UALCAN for differential analysis, which found that SPOCK2 was significantly associated with clinical features on transcriptional and translational levels. As shown in Fig. 3A-E, SPOCK2 mRNA expression level was significantly correlated with patient's race (Caucasian vs. African-american, $P<0.01$ ) and tumor grade (Grade 2 vs. Grade $3, P<0.05$ ), and has no relation with patient's age, individual cancer stage, and TP53 mutation status $(P>0.05)$. The univariate 
analysis with logistic regression showed that upregulated SPOCK2 mRNA expression is positively associated with tumor residual ( $\mathrm{OR}=1.746$ for RD vs. NRD, $P=0.045$ ) (Table 1). Furthermore, we found that protein expression level of SPOCK2 was positively associated with tumor grade (Normal vs. Grade 2, $P<0.001$; Normal vs. Grade 3, $P<0.01$ ), patient's race (Caucasian vs. Asian, $P<0.01$ ), and individual cancer stage (Stage 1 vs. Stage $4, P<0.01$; Stage 3 vs. Stage $4, P<0.001$ ) (Fig. 3 F-H). These results revealed that overexpressed SPOCK2 was susceptible to a more advanced disease and was more common in the Caucasian patients, indicating that SPOCK2 has potentials to be a prognostic indicator for HGSOC patients.

\section{High SPOCK2 expression is associated with poor prognosis}

Kaplan-Meier curves were used to explore the relationships between SPOCK2 mRNA expression and OS in HGSOC patients. As shown in Fig. 4A, the result of Kaplan-Meier analysis showed that HGSOC patients with high level of SPOCK2 mRNA expression in the TCGA-OV data set had a worse OS than those with a low expression ( $\mathrm{HR}=1.45, \mathrm{Cl}$ : 1.12-1.88, $P=0.005)$. Next, we performed subgroup survival analyses of OS based on clinical characteristics, which exhibited that prognosis of patients with high-level SPOCK2 expression was significantly poor in the White $(\mathrm{HR}=1.44, \mathrm{Cl}: 1.09-1.89, P=0.01)$, stage III\&IV $(\mathrm{HR}=1.48$, Cl: $1.14-1.92, P=0.004)$, and Grade $3 \& 4$ (HR=1.47, Cl: $1.11-1.95, P=0.007)$ subgroups of OS (Fig. 4D-I). Notably, for HGSOC cases with high-level SPOCK2 expression, patients aged over 60 years $(\mathrm{HR}=1.66, \mathrm{Cl}$ : $1.15-2.41, P=0.007)$ and under 60 years $(\mathrm{HR}=1.47, \mathrm{Cl}: 1.02-2.12, P=0.039)$ both have comparatively worse OS than those with low-level expression (Fig. 4B-C). To further find clinical factors associated with survival, we first performed univariate logistic regression analysis which found that age $(H R=1.355, \mathrm{Cl}$ : 1.046-1.754, $P=0.021)$, primary therapy outcome ( $\mathrm{HR}=0.301, \mathrm{Cl}: 0.204-0.444, P<0.001)$, tumor status $(\mathrm{HR}=9.576, \mathrm{Cl}: 4.476-20.486, P<0.001)$, tumor residual $(\mathrm{HR}=2.313, \mathrm{Cl}: 1.486-3.599, P<0.001)$, and SPOCK2 expression level $(\mathrm{HR}=1.445, \mathrm{Cl}: 1.115-1.872, P=0.005)$ were significantly associated with OS (Fig. 5A, Supplementary Table 1). In addition, multivariate Cox regression analysis showed that primary therapy outcome (HR=0.365, Cl: $0.237-0.563, P<0.001)$ and tumor status (HR=19.162, Cl: 4.68978.307, $P<0.001)$ were independent prognostic factors of OS for HGSOC; SPOCK2 expression level, however, was not still an independent factor related to OS (HR=1.297, Cl: $0.939-1.791, P=0.114)$ (Fig. 5B, Supplementary Table 1).

\section{Constructing the prognostic models for HGSOC}

To quantitatively verify that SPOCK2 mRNA is an advisable approach predicting the prognosis of HGSOC patients, SPOCK2 and other clinicopathological parameters including age, FIGO stage, histological grade, and primary therapy outcome were used to construct a prediction model for OS. We, therefore, established a nomogram of OS based on multivariate Cox regression analysis to assign points to the abovementioned variables. The points assigned to these variables were accumulated and recorded as the total score. For example, HGSOC patients with high SPOCK2 mRNA expression (25 points), advanced FIGO stages (59 points), and age over 60 years (19 points) received a total score of 103 . Then, we draw a vertical line directly down from the total points axis at scale of 103 to the survival probability axis. Therefore, the 1-, 3-, and 5-year survival probabilities were $>95 \%, 68 \%$, and $36 \%$, respectively (Fig. $6 \mathrm{~A}$ ). 
Moreover, we evaluated the performance of the nomogram by using Calibration curves, and the result showed that C-index of the model was 0.638 (Cl: 0.616-0.661), which indicated that the prediction efficiency of this model is moderately accurate (Fig. 6B). Overall, these results suggested that the nomogram was a better model for predicting survival in HGSOC patients than individual prognostic factors.

\section{Co-expressed genes of SPOCK2 in HGSOC}

To explore the co-expressed genes of SPOCK2, we used the LinkFinder module. As shown in the Volcano plot (Fig. 7B), a total of 6,453 genes were identified to be significantly correlated with SPOCk2 $(P<0.05)$. Following this, we showed top 50 genes that positively and negatively correlated with SPOCk2 in the heat maps, respectively (Fig. 7A and 7D). In addition, the scatter plots were used to present the most closely correlated genes with SPOCK2, including CRB2 (Pearson-Correlation: 0.606, $P=8.543 \mathrm{E}-32$ ), ARHGAP23 (Pearson-Correlation: 0.533, $P=1.247 \mathrm{E}-23$ ), TGM1 (Pearson-Correlation: 0.532, $P=1.518 \mathrm{E}-23$ ), AKAP7 (Pearson-Correlation: $-0.377, P=1.191 \mathrm{E}-11$ ), and MERTK (Pearson-Correlation: $-0.361, P=9.034 \mathrm{E}-11$ ) (Fig. 7C and 7E-H).

\section{Functional enrichment analysis of SPOCK2}

We used the LinkInterpreter module to explore functional enrichment information of SPOCK2 that contribute to tumorigenesis or tumor progression of HGSOC. KEGG pathway analysis showed that SPOCK2-correlated genes were mainly involved in focal adhesion, leukocyte transendothelial migration, and ECM-receptor interaction (Fig. 8A). GO_BP (biological process) was mainly associated with cell junction organization, cell-cell adhesion via plasma-membrane adhesion molecules, and extracellular structure organization (Fig. 8B). GO_CC (cell component) was mainly related with extracellular matrix, actin cytoskeleton, contractile fiber, and cell-substrate junction (Fig. 8C). GO_MF (molecular function) was mainly associated with extracellular matrix structure constituent, extracellular matrix binding, actin binding, cell adhesion mediator activity, cell adhesion molecule binding, and growth factor binding (Fig. 8D). Overall, these top KEGG and GO enrichment items suggested that SPOCK2 might play an important role in modulating extracellular matrix.

\section{Immune infiltration analysis of SPOCK2}

Given the fact that immune infiltration is deeply involved in anti-tumor responses, we investigated the relationships between expression level of SPOCK2 mRNA and abundance of TILs. First, the heat map showed Spearman correlations between 28 kinds of immune cells and SPOCK2 mRNA expression in 30 TCGA cancer types, which was downloaded from TISIDB (Fig. 9A). For HGSOC, correlations between 24 TIL types and SPOCK2 were analyzed by using SSGSEA and the Spearman method (Fig. 9B). The scatter plots indicated that SPOCK2 mRNA expression positively correlated with infiltration of natural killer (NK) cells $(R=0.310, P<0.001)$, T effector memory (Tem) cells $(R=0.220, P<0.001)$, T central memory (Tcm) cells $(R=0.190, P<0.001)$, and Mast cells $(R=0.110, P=0.033)$; by contrast, SPOCK2 mRNA expression negatively correlated with infiltration of activated dendritic cells (aDC) $(R=-0.170, P=0.001)$ and T helper 2 (Th2) cells $(R=-0.140, P=0.005)(F i g .9 C-H)$. In addition, the Wilcoxon rank sum test was applied to 
further verify the correlations between SPOCK2 expression and abovementioned TILs. The plots confirmed that patients in SPOCK2-high group had more NK cells $(P<0.001)$, Tem cells $(P<0.01)$, and Tcm cells $(P<0.05)$, and had less aDC $(P<0.01)$ and Th2 cells $(P<0.01)$; however, the plot showed no significant difference of Mast cells infiltrating abundance between SPOCK2-high and -low groups $(P>$ 0.05), which is not consistent with the result of scatter plot (Fig. 10). These results manifested that SPOCK2 might have double-edged functions in coordinating the induction of TILs.

\section{Discussion}

In this study, we found that both mRNA and protein expression of SPOCK2 were upregulated in ovarian serous cystadenocarcinoma along with a high expression of SPOCK2 mRNA in the most TCGA cancers. Further ROC curve analysis confirmed that SPOCK2 could be a promising diagnostic biomarker to distinguish HGSOC from benign tissues. To investigate factors that influence SPOCK expression, subgroup analyses based on clinical variables were done in all patients enrolled in the UALCAN dataset, suggesting that SPOCK2 expression is correlated with patient's race, tumor grade, and cancer stage. A critical element of this study is the evaluation of the effect of SPOCK2 on OS by using Kaplan-Meier and univariate logistic regression analyses, in which high SPOCK2 mRNA expression is associated with a shorter OS. Additionally, functional enrichment analysis of SPOCK2-correlated genes revealed that SPOCK2 may play a specific role in regulating functions of extracellular matrix. Exploratory analyses of TILs were conducted to examine the role of SPOCK2 in immune infiltration, showing that there were significant associations between SPOCK2 mRNA expression level and TILs abundance. Overall, this study has led to an improved understanding of SPOCK2 in HGSOC and provided potential therapeutic target.

SPOCK family comprised of SPOCK1, SPOCK2, and SPOCK3, and was originally identified by CDNA cloning, with amino acid homologies (Bradshaw et al. 2012; Vannahme et al. 1999). SPOCK proteins share a unique $\mathrm{N}$-terminal region, followed by a follistatin-like (FS) domain, calcium-binding (EC) domain, thyroglobulin type-1 (TY) domain, and a C-terminal tail encompassing heparan/chondroitin sulfate glycosaminoglycan attachment sites at two serine residues (Alliel et al. 1993; Bonnet et al. 1996; Krajnc et al. 2020). To connect protein functions to the spatial organization of SPOCK2 protein domains, Krajnc et al. confirmed that the FS-EC-TY domain triplet formed a structure core and was responsible for executing functions (Krajnc et al. 2020). All three SPOCKs are extracellular proteoglycans highly expressed in brain, ovary, lung, testis, prostate, and kidney, and it is expected that SPOCKs might contribute to extracellular matrix and associate with tumor progression (Alliel et al. 1993; Bonnet et al. 1996; Vannahme et al. 1999). To gain insight into the biological functions of SPOCKs in human cancers, we focused on SPOCK2 as a presentative of the family because of its upregulated expression and prognostic potential in HGSOC patients (Ren et al. 2011). Nordgard et al. first described the correlation of SPOCK2 with human cancer and found that oligonucleotide polymorphism of SPOCK2 gene was associated with deletion of $16 \mathrm{q}$ chromosome in breast cancer (Nordgard et al. 2008). Encoded by the SPOCK2 gene, it is a 424-amino acid protein with a C-terminal tail that binds to glycosaminoglycans to form proteoglycans (Vannahme et al. 1999). In addition to being structural components of extracellular matrix, proteoglycans can serve as important biochemical signals that regulate cell proliferation, differentiation and migration, and modulate 
vascular networks and immune cell infiltration (Gordon-Weeks and Yuzhalin 2020). Thus, it is essential to determine whether abnormal changes of SPOCK2 exist in HGSOC and how its properties influence tumor progression.

By analyzing the expression level of SPOCK2 in HGSOC, we noted that both mRNA and protein level of SPOCK2 in HGSOC were significantly higher than those in normal ovary tissues, which were influenced by clinical features such as patient's race, tumor grade, and cancer stage. Few studies have been conducted to explore the underlying reasons of why these clinical characteristics could change genetic performance of SPOCK2. Nevertheless, some studies have focused on mechanisms of up- and down-regulated expression of SPOCK2 and concluded that promoter methylation of SPOCK2 gene may be one of reasons for abnormal expression of SPOCK2. Ren et al. carried out a retrospective study to compare the protein expression level and methylation status of SPOCK2 in malignant ovarian tissues and benign tissues (Ren et al. 2011). They found that the frequency of SPOCK2 hypermethylation in malignant ovarian tissues (45\%) was significantly higher than $6.7 \%$ in the benign tissues; synchronously, the loss rate of SPOCK2 protein in malignant ovarian tissues was $44 \%$, which was significantly higher than $13.3 \%$ in the benign tissues, indicating that SPOCK2 hypermethylation might lead to loss expression of protein. Alternatively, Chung et al. verified that treatment with 5-aza-2'-deoxycytidine, a demethylating agent, could restore expression of SPOCK2, suggesting that DNA methylation is related to transcriptional silencing of SPOCK2 in prostate, colon, and breast cancer (Chung et al. 2008). In addition to DNA methylation, future researches could focus on other epigenetic modifications to figure out how SPOCK2 expression in HGSOC is upregulated.

SPOCK2 was highly expressed in HGSOC patients and was related to poorer OS. This study has, therefore, characterized SPOCK2 mRNA expression as a prognostic biomarker for prognosis. On the contrary, Lu et al. found that overexpressed SPOCK2 was significantly associated with better OS (HR = $0.302, P=0.016$ ) in patients with pancreatic ductal adenocarcinoma (Lu et al. 2021). Similarly, an in vitro experiment revealed that overexpression of SPOCK2 contribute to inhibit cell proliferation and invasion in endometrium-derived cancer cell lines. The researchers also found that inhibitory functions of SPOCK2 on tumor cell progression were achieved partly through suppressing expression or activation of MT1MMP and MMP2 (Ren et al. 2020). However, another in vitro study confirmed that abundant distribution of SPOCK2 may contribute to glioma invasion by countering the inhibitory action of SPOCK1 and SPOCK3 and supporting MT-MMP-mediated cell migration (Nakada et al. 2003). In summary, these findings suggested that functions of SPOCKs may differ among human cancers. Although SPOCK2 protein was upregulated in HGSOC, high expression of SPOCK2 protein failed to be a prognostic indicator in this study. Considering that SPOCK2 is a potential biomarker for OS, a nomogram was constructed to predict 1-, 3-, and 5-year survival possibility. Our findings indicated that this nomogram model could help to differentiate more advanced HGSOC patients and determine more aggressive therapeutic regimens.

Functional enrichment analyses in this study demonstrated that biological processes of SPOCK2 in HGSOC often require gene network interactions. To further understand biological capabilities of SPOCK2 in HGSOC, correlated genes were used to perform functional enrichment analysis, finding that SPOCK2 
may participate in extracellular matrix structure constituent, focal adhesion, cell junction organization, and growth factor binding. According to the results of previous studies, degradation of extracellular matrix surrounding focal adhesion sites played an important role in regulating metastatic process, which required the proteolytic activity of MT1-MMP. Researcher further verified that focal adhesion kinase (FAK), a focal adhesion component, was essential to recruit MT1-MMP molecules (Wang and McNiven 2012). Moreover, Nakada et al. found that SPOCK2 could abolish inactivation of MT1-MMP and MT3-MMP by other SPOCK family members and permit migration of glioma cells in the presence of SPOCK1 and SPOCK3 proteins (Nakada et al. 2003). Accordingly, SPOCK2 could promote remodeling of extracellular matrix and, therefore, was considered as an important molecule in cell migration. Overall, SPOCK 2 played critical role in tumor development, suggesting that more future researches would be needed to explore properties of SPOCK2 in HGSOC.

During cancer progression, tumor cells can establish an overtly immunosuppressive tumor microenvironment that is composed of infiltrating immune cells, endothelial cells, and fibroblasts, often embedded within extracellular matrix (Anderson et al. 2017). Numerous studies indicated that abundance of tumor infiltrating immune cells is closely correlated with clinical survival (Hao et al. 2020a; Hao et al. 2020b). However, extracellular matrix surrounding the tumor islets can integrate and deliver multiple complex signals to leukocytes that affect their biological functions, thereby indicating that the complex relationships between extracellular matrix and TILs should be addressed (Pickup et al. 2014).

Extracellular matrix can exert antineoplastic activity through providing migratory highways on which leukocytes can directly invade into inflammation-inflamed tumor areas in response to chemo-attractant matrix molecules released via MMP-mediated degradation (Pickup et al. 2014; Sorokin et al. 2010). In this regard, SPOCK2 could promote leukocyte migration and activate anti-tumor inflammation because its facilitative role of MT-MMP-mediated proteolysis that releases chemo-attractant fragments. Similarly, this study found that SPOCK2 positively correlated with infiltration of NK cells, Tem cells, Tcm cells, and Mast cells, indicating a pro-immunogenic role of SPOCK2. Besides inducing anti-tumor immunity, extracellular matrix can directly inhibit $T$ cell activity through impairing the process of antigen presentation (Meyaard et al. 2008; Vesely et al. 2011). Coincidentally, we found that SPOCK2 negatively correlated with infiltration of aDC which is the most capable antigen-presenting cells, suggesting a pro-tumoral role of SPOCK2. On the basis of our findings and previous studies, we concluded that effects of abnormal SPOCK2 expression on TILs still remain elusive, appealing to more fundamental researches.

In conclusion, this study provided evidence for upregulated expression of SPOCK2 and found that SPOCK2 had diagnostic and prognostic potential for HGSOC. In addition to explorations of SPOCK2related pathways that indicated a critical role in remodeling extracellular matrix, immune cell infiltration analyses provided new understandings for associations between SPOCK2 and TILs. Although a lack of in vivo/vitro experiments and use of public databases make it difficult to detail the mechanisms of SPOCK2 that contribute HGSOC progression, this article is expected to provide a new direction for predicting prognosis and exploring therapeutic targets. In the future, more fundamental experiments with clinical samples instead of data mining should be well-designed. 


\section{Abbreviations}

HGSOC: High-grade serous ovarian cancer; EOC: Epithelial ovarian cancer; PARPi: Poly (ADP-ribose) polymerase inhibitors; SPOCK2: SPARC (osteonectin), cwCV and kazal-like domains proteoglycan 2; MMP2: Matrix metalloproteinase 2; MT1-MMP: Membrane-type 1 matrix metalloproteinase; CTLA-4: Cytotoxic T-lymphocyte-associated antigen 4; TCGA: The Cancer Genome Atlas; GTEx: Genotype Tissue Expression; KEGG: Kyoto encyclopedia of genes and genomes; GO: Gene ontology; HTSeq-FPKM: High throughput sequencing-fregments per kilobase per million; TPM: transcription per million reads; HPA: The human protein atlas; CPTAC: Clinical proteomic tumor analysis consortium; ROC: Receptor operating characteristic; C-index: Concordance index; GSEA: Gene set enrichment analysis; TILs: Tumor-infiltrating lymphocytes; BRCA: Breast infiltrating carcinoma; $\mathrm{CHOL}$ : cholangiocarcinoma; DLBCL: Diffuse large B cell lymphoma; ESCA: Esophageal carcinoma; HNSC: Head and neck squamous cell carcinoma; LAML: Acute myeloid leukemia; LGG: Brain low grade glioma; LIHC: Liver hepatocellular carcinoma; OV: Ovarian serous cystadenocarcinoma; PAAD: Pancreatic cancer; PCPG: Pheochromocytoma and paraganglioma; SKCM: Skin melanoma; STAD: Gastric cancer; THCA: Thyroid cancer; THYM: Thymic cancer; UCEC:

Endometrial cancer; UCS: Uterine sarcoma; ACC: Drenocortical carcinoma; COAD: Colon adenocarcinoma; GBM: Pleomorphic glioma; KICH: Renal chromophobe cell carcinoma; KIRC: Renal clear cell carcinoma; KIRP: Renal papillary cell carcinoma; LUAD: lung adenocarcinoma; LUSC: Lung squamous cell carcinoma; READ: Rectum adenocarcinoma; TGCT: Testicular germ cell tumors; AUC: Area under the curve; BP: Biological process; CC: Cell component; MF: Molecular function; NK: Natural killer; Tem: T effector memory; Tcm: T central memory; aDC: Activated dendritic cells; Th2: T helper 2; FS: Follistatin-like; EC: Calcium-binding; TY: Thyroglobulin type-1; FAK: Focal adhesion kinase.

\section{Declarations}

\section{ETHICS APPROVAL AND CONSENT TO PARTICIPATE}

The study was approved by the Research Ethics Committee of the First Affiliated Hospital of Xi'an Jiaotong University. All patients signed written informed consent before sample collection at the First Affiliated Hospital of Xi'an Jiaotong University

\section{CONSENT FOR PUBLICATION}

All authors read and agreed to the content of the final manuscript, and consented to publish this study.

\section{AVAILABILITY OF DATA AND MATERIALS}

Publicly available datasets were analyzed in this study. This data can be found here: TCGA-OV cohort https://genome-cancer.ucsc.edu/. The raw data supporting the conclusions of this study will be made available by the corresponding authors, without undue reservation. 
The authors declare that they have no known competing financial interests or personal relationships that could have appeared to influence the results of this study.

\section{FUNDING}

This work was supported by the National Natural Science Foundation of China (grant No. 81101949) and the Shaanxi Natural Science Basic Research Program-General Projects (grant No. 2021JM-282).

\section{AUTHOR CONTRIBUTIONS}

Jiatao Hao, Yumeng Cao, and Ruifang An contributed to the conception and design of this study. Yumeng Cao, Xiaoya Gu, Minghai Ma, Weihong Wang, and Yan Xue participated in data collection, data analysis, and data interpretation. Jiatao Hao was responsible for manuscript writing. Ruifang An edited the draft of the manuscript. All authors contributed to the article and approved the submitted version.

\section{ACKNOWLEDGMENTS}

We thank Meng Li (Baoji Central Hospital, Baoji, China) for his help and valuable suggestion.

\section{References}

1. Alliel PM, Perin JP, Jollès P, Bonnet FJ. Testican, a multidomain testicular proteoglycan resembling modulators of cell social behaviour. Eur J Biochem. 1993;214(1):347- 50.

2. Anderson KG, Stromnes IM, Greenberg PD. Obstacles Posed by the Tumor Microenvironment to T cell Activity: A Case for Synergistic Therapies. Cancer Cell. 2017;31(3):311-25.

3. Bonnet F, Périn JP, Charbonnier F, Camuzat A, Roussel G, Nussbaum JL, et al. Structure and cellular distribution of mouse brain testican. Association with the postsynaptic area of hippocampus pyramidal cells. J Biol Chem. 1996;271(8):4373- 80.

4. Bradshaw AD. Diverse biological functions of the SPARC family of proteins. Int J Biochem Cell Biol. 2012;44(3):480-8.

5. Chandrashekar DS, Bashel B, Balasubramanya SAH, Creighton CJ, Ponce-Rodriguez I, Chakravarthi BVSK, et al. UALCAN: A Portal for Facilitating Tumor Subgroup Gene Expression and Survival Analyses. Neoplasia. 2017;19(8):649-58.

6. Chung W, Kwabi-Addo B, Ittmann M, Jelinek J, Shen L, Yu Y, et al. Identification of novel tumor markers in prostate, colon and breast cancer by unbiased methylation profiling. PLoS One. 2008;3(4):e2079.

7. Crespo A, García-Suárez O, Fernández-Vega I, Solis-Hernandez MP, García B, Castañón S, et al. Heparan sulfate proteoglycans undergo differential expression alterations in left sided colorectal cancer, depending on their metastatic character. BMC Cancer. 2018;18(1):687.

8. Edwards NJ, Oberti M, Thangudu RR, Cai S, McGarvey PB, Jacob S, et al. The CPTAC Data Portal: A Resource for Cancer Proteomics Research. J Proteome Res. 2015;14(6):2707-13. 
9. Einzig Al, Wiernik PH, Sasloff J, Runowicz CD, Goldberg GL. Phase II study and long- term follow-up of patients treated with taxol for advanced ovarian adenocarcinoma. J Clin Oncol. 1992;10(11):174853.

10. González-Martín A, Pothuri B, Vergote I, DePont Christensen R, Graybill W, Mirza MR, et al. Niraparib in Patients with Newly Diagnosed Advanced Ovarian Cancer. N Engl J Med. 2019;381(25):2391-402.

11. Gordon-Weeks A, Yuzhalin AE. Cancer Extracellular Matrix Proteins Regulate Tumour Immunity. Cancers (Basel). 2020;12(11):3331.

12. Hamanishi J, Mandai M, Ikeda T, Minami M, Kawaguchi A, Murayama T, et al. Safety and Antitumor Activity of Anti-PD-1 Antibody, Nivolumab, in Patients With Platinum-Resistant Ovarian Cancer. J Clin Oncol. 2015;33(34):4015-22.

13. Hao J, Liu Y, Zhang T, He J, Zhao H, An R, et al. Efficacy and safety of PARP inhibitors in the treatment of advanced ovarian cancer: An updated systematic review and meta-analysis of randomized controlled trials. Crit Rev Oncol Hematol. 2021;157:103145.

14. Hao J, Li M, Zhang T, Yu H, Liu Y, Xue Y, et al. Prognostic Value of Tumor-Infiltrating Lymphocytes Differs Depending on Lymphocyte Subsets in Esophageal Squamous Cell Carcinoma: An Updated Meta-Analysis. Front Oncol. 2020a;10:614.

15. Hao J, Yu H, Zhang T, An R, Xue Y. Prognostic impact of tumor-infiltrating lymphocytes in high grade serous ovarian cancer: a systematic review and meta- analysis. Ther Adv Med Oncol. 2020b;12:1758835920967241.

16. Krajnc A, Gaber A, Lenarčič B, Pavšič M. The Central Region of Testican-2 Forms a Compact Core and Promotes Cell Migration. Int J Mol Sci. 2020;21(24):9413.

17. Kubalanza K, Konecny GE. Mechanisms of PARP inhibitor resistance in ovarian cancer. Curr Opin Obstet Gynecol. 2020;32(1):36-41.

18. Kurnit KC, Fleming GF, Lengyel E. Updates and New Options in Advanced Epithelial Ovarian Cancer Treatment. Obstet Gynecol. 2021;137(1):108-21.

19. Kuroki L, Guntupalli SR. Treatment of epithelial ovarian cancer. BMJ. 2020;371:m3773.

20. Lheureux S, Braunstein M, Oza AM. Epithelial ovarian cancer: Evolution of management in the era of precision medicine. CA Cancer J Clin. 2019;69(4):280- 304.

21. Liu G, Ren F, Song Y. Upregulation of SPOCK2 inhibits the invasion and migration of prostate cancer cells by regulating the MT1-MMP/MMP2 pathway. PeerJ. 2019;7:e7163.

22. Lu Q, Zhang Y, Chen X, Gu W, Ji X, Chen Z. Prognostic significance and immune infiltration of microenvironment-related signatures in pancreatic cancer. Medicine (Baltimore). 2021;100(12):e24957.

23. Mirza MR, Monk BJ, Herrstedt J, Oza AM, Mahner S, Redondo A, et al. Niraparib Maintenance Therapy in Platinum-Sensitive, Recurrent Ovarian Cancer. N Engl J Med. 2016;375(22):2154-64.

24. Meyaard L. The inhibitory collagen receptor LAIR-1 (CD305). J Leukoc Biol. 2008;83(4):799-803. 
25. Nakada M, Miyamori H, Yamashita J, Sato H. Testican 2 abrogates inhibition of membrane-type matrix metalloproteinases by other testican family proteins. Cancer Res. 2003;63(12):3364-9.

26. Nordgard SH, Johansen FE, Alnaes GI, Bucher E, Syvänen AC, Naume B, et al. Genome-wide analysis identifies $16 \mathrm{q}$ deletion associated with survival, molecular subtypes, mRNA expression, and germline haplotypes in breast cancer patients. Genes Chromosomes Cancer. 2008;47(8):680-96.

27. Oza AM, Matulonis UA, Malander S, Hudgens S, Sehouli J, Del Campo JM, et al. Quality of life in patients with recurrent ovarian cancer treated with niraparib versus placebo (ENGOT-OV16/NOVA): results from a double-blind, phase 3, randomised controlled trial. Lancet Oncol. 2018;19(8):1117-25.

28. Pickup MW, Mouw JK, Weaver VM. The extracellular matrix modulates the hallmarks of cancer. EMBO Rep. 2014;15(12):1243-53.

29. Pujade-Lauraine E. New treatments in ovarian cancer. Ann Oncol. 2017;28(suppl_8):viii57-viii60.

30. Reid BM, Permuth JB, Sellers TA. Epidemiology of ovarian cancer: a review. Cancer Biol Med. 2017;14(1):9-32.

31. Ren F, Wang D, Wang Y, Chen P, Guo C. SPOCK2 Affects the Biological Behavior of Endometrial Cancer Cells by Regulation of MT1-MMP and MMP2. Reprod Sci. 2020;27(7):1391-9.

32. Ren F, Wang DB, Li T. Epigenetic inactivation of SPOCK2 in the malignant transformation of ovarian endometriosis. Zhonghua Fu Chan Ke Za Zhi. 2011;46(11):822-5.

33. Ru B, Wong CN, Tong Y, Zhong JY, Zhong SSW, Wu WC, et al. TISIDB: an integrated repository portal for tumor-immune system interactions. Bioinformatics. 2019;35(20):4200-2.

34. Said NA, Elmarakby AA, Imig JD, Fulton DJ, Motamed K. SPARC ameliorates ovarian cancerassociated inflammation. Neoplasia. 2008;10(10):1092-104.

35. Sorokin L. The impact of the extracellular matrix on inflammation. Nat Rev Immunol. 2010;10(10):712-23.

36. Vannahme C, Schübel S, Herud M, Gösling S, Hülsmann H, Paulsson M, et al. Molecular cloning of testican-2: defining a novel calcium-binding proteoglycan family expressed in brain. J Neurochem. 1999;73(1):12-20.

37. Vasaikar SV, Straub P, Wang J, Zhang B. LinkedOmics: analyzing multi-omics data within and across 32 cancer types. Nucleic Acids Res. 2018;46(D1):D956-D963.

38. Vesely MD, Kershaw MH, Schreiber RD, Smyth MJ. Natural innate and adaptive immunity to cancer. Annu Rev Immunol. 2011;29:235-71.

39. Vivian J, Rao AA, Nothaft FA, Ketchum C, Armstrong J, Novak A, et al. Toil enables reproducible, open source, big biomedical data analyses. Nat Biotechnol. 2017;35(4):314-6.

40. Wang Y, McNiven MA. Invasive matrix degradation at focal adhesions occurs via protease recruitment by a FAK-p130Cas complex. J Cell Biol. 2012;196(3):375-85.

\section{Tables}

Due to technical limitations, table 1 is only available as a download in the Supplemental Files section. 


\section{Figures}

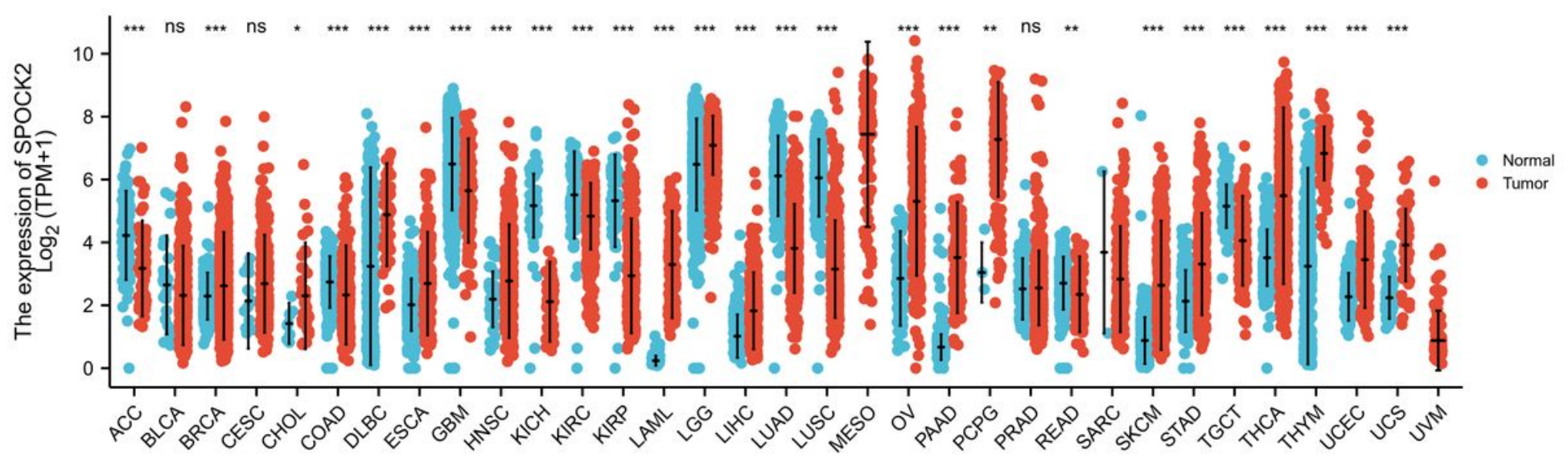

\section{Figure 1}

Transcription level of SPOCK2 in pan-cancer perspective. ACC, drenocortical carcinoma; BRCA, breast infiltrating carcinoma; $\mathrm{CHOL}$, cholangiocarcinoma; $\mathrm{COAD}$, colon adenocarcinoma; $\mathrm{DLBCL}$, diffuse large $\mathrm{B}$ cell lymphoma; ESCA, esophageal carcinoma; GBM, pleomorphic glioma; HNSC, head and neck squamous cell carcinoma; $\mathrm{KICH}$, renal chromophobe cell carcinoma; KIRC, renal clear cell carcinoma; KIRP, renal papillary cell carcinoma; LAML, acute myeloid leukemia; LGG, brain low grade glioma; LIHC, liver hepatocellular carcinoma; LUAD, lung adenocarcinoma; LUSC, lung squamous cell carcinoma; OV, ovarian serous cystadenocarcinoma; PAAD, pancreatic cancer; PCPG, pheochromocytoma and paraganglioma; READ, rectum adenocarcinoma; SKCM, skin melanoma; STAD, gastric cancer; TGCT, testicular germ cell tumors; THCA, thyroid cancer; THYM, thymic cancer; UCEC, endometrial cancer; UCS, uterine sarcoma. (Significant codes of $P$-value: $n s, P \geq 0.05 ; *, P<0.05 ; \star \star, P<0.01 ; * \star \star, P<0.001$ ). 

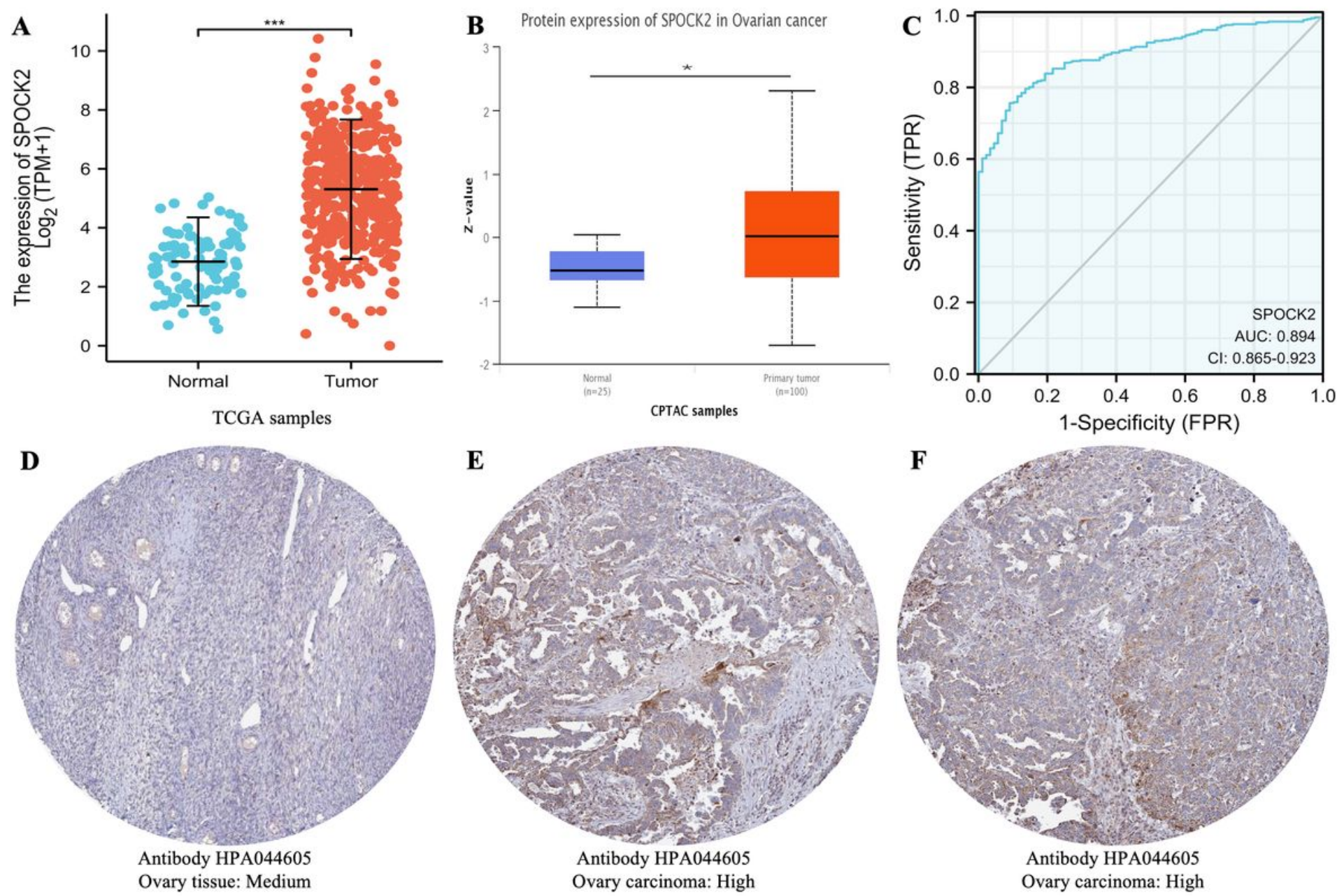

Figure 2

Expression profile and diagnostic performance of SPOCK2 in high-grade serous ovarian cancer (HGSOC). Expression level of SPOCK2 mRNA (A). Expression level of SPOCK2 protein (B). Receptor operating characteristic (ROC) curve for SPOCK2 (C). Protein levels of SPOCK2 in ovary tissue and ovary carcinoma

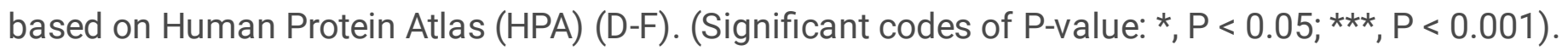




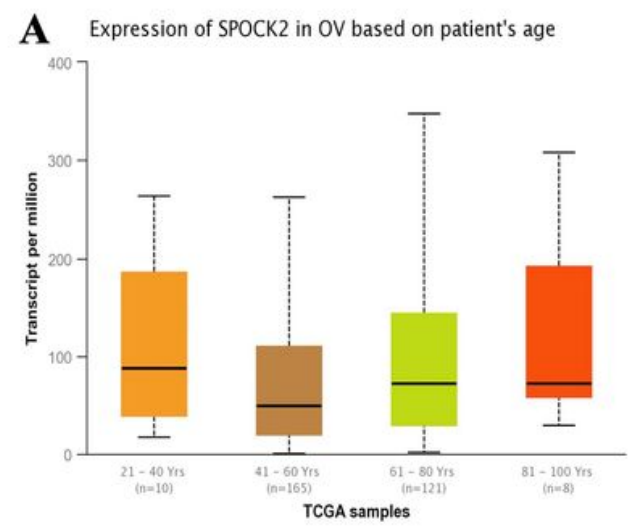

D Expression of SPOCK2 in OV based on individual cancer stages

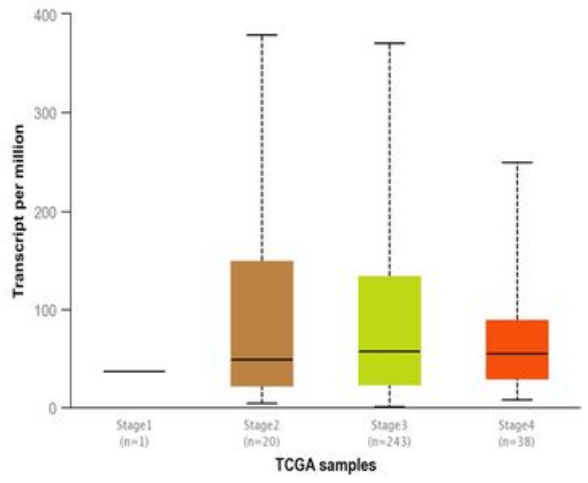

G

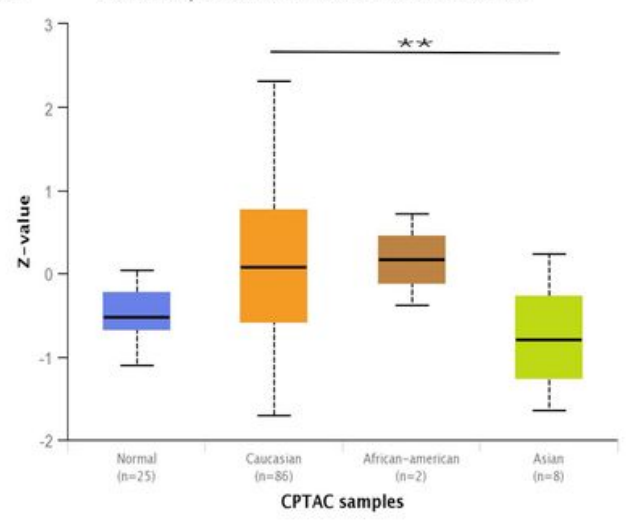

B Expression of SPOCK2 in OV based on patient's race

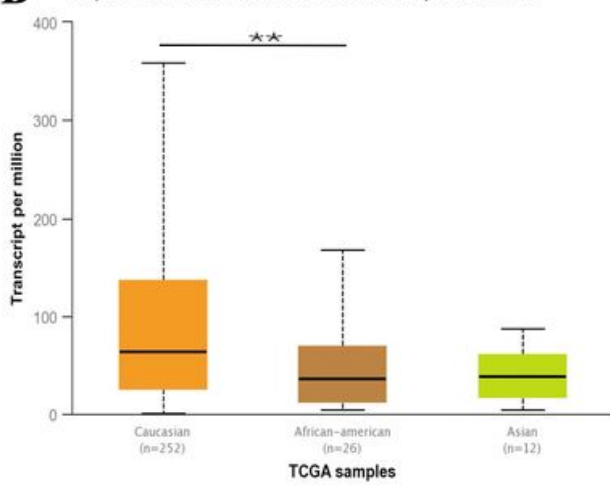

E Expression of SPOCK2 in OV based on TP53 mutation status

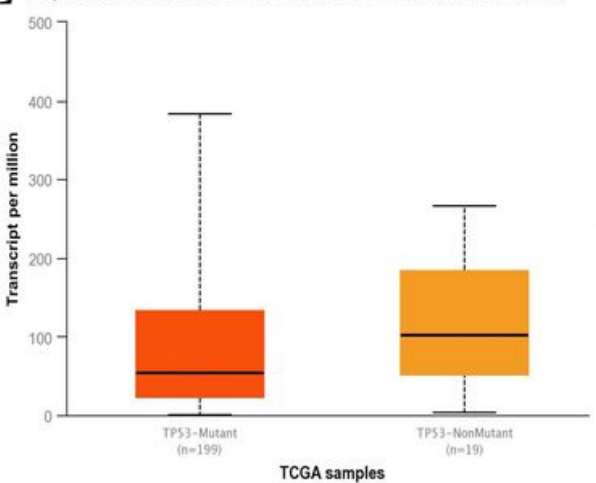

H Protein expression of SPOCK2 in Ovarian cancer

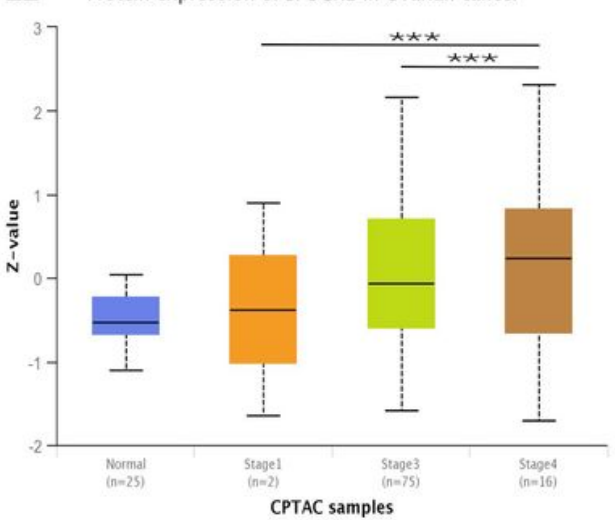

C Expression of SPOCK2 in OV based on tumor grade

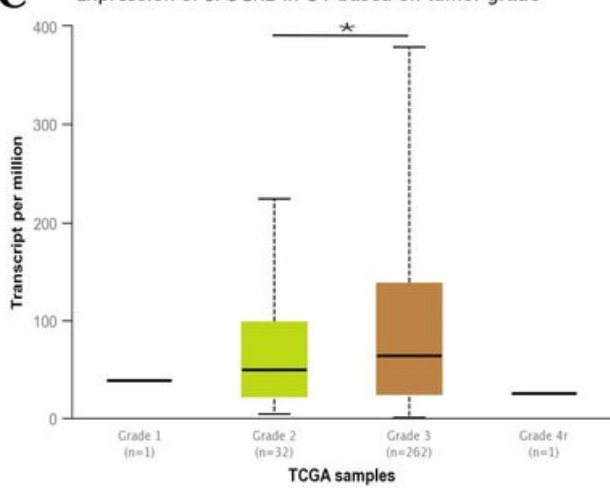

F Protein expression of SPOCK2 in Ovarian cancer

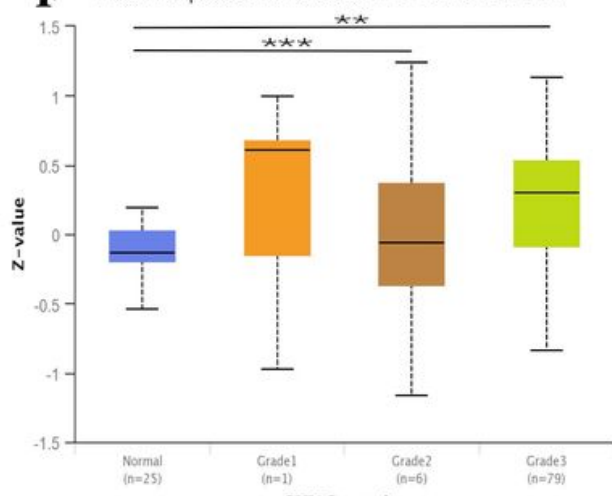

CPTAC samples

Figure 3

SPOCK2 expression level in subgroups of patients with HGSOC. Boxplots of SPOCK2 mRNA expression based on patient's age (A), patient's race (B), tumor grade (C), cancer stage (D), and TP53 mutation status (E). Boxplots of SPOCK2 protein expression based on tumor grade $(F)$, patient's race $(G)$, and cancer stage $(H)$. (Significant codes of P-value: *, $P<0.05 ; * \star \star, P<0.001$ ). 

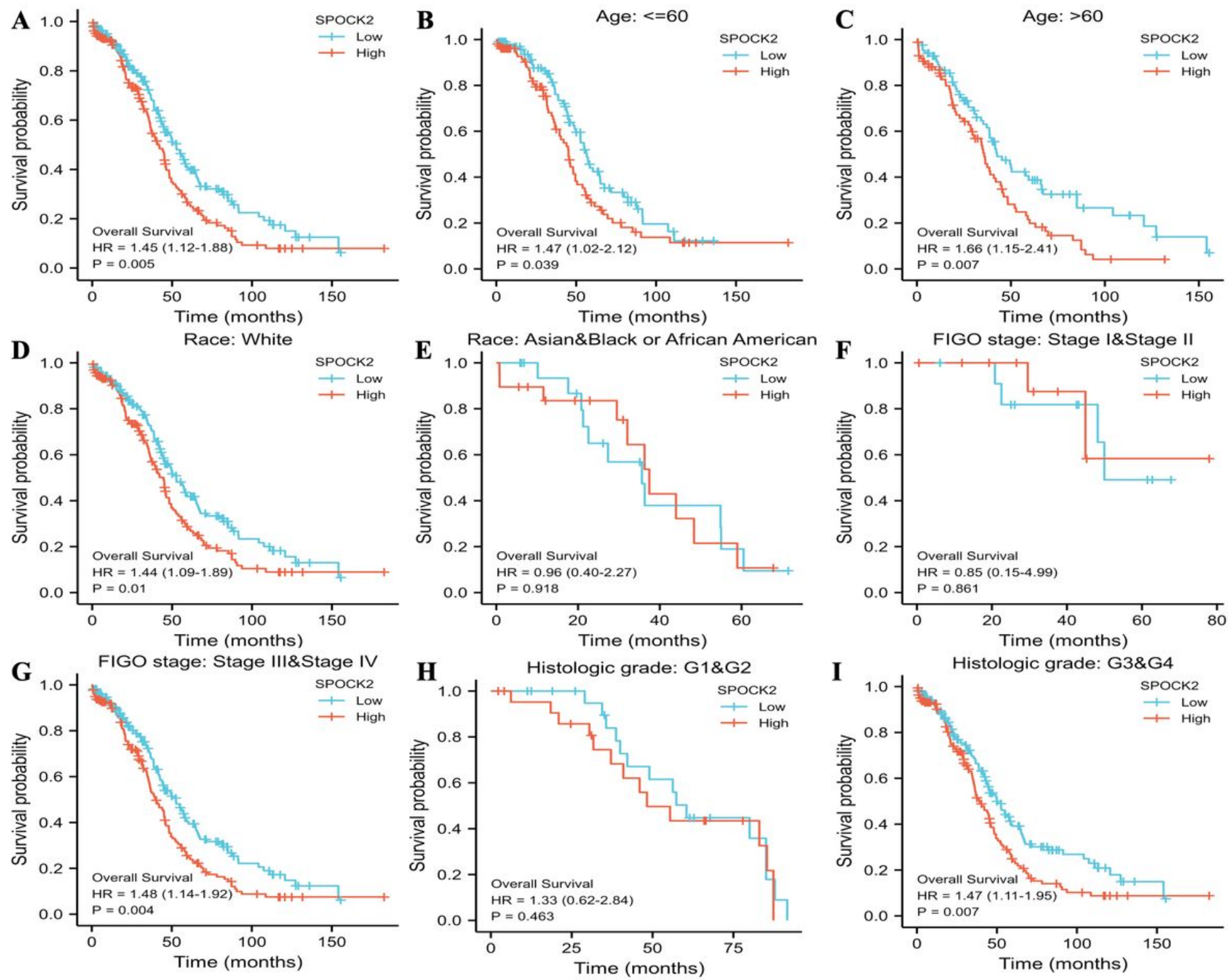

\section{Figure 4}

Kaplan-Meier survival curves comparing the high and low expression of SPOCK2 in HGSOC. Survival curve of OS between SPOCK2-high and -low patients (A). Survival curves of OS between SPOCK2-high and -low subgroups among patients with age $\leq 60$ (B), age $₫ 60$ (C), White (D), Asian \& Black or African American (E), stage I\&II (F), stage III\&IV (G), Grade 1\&2 (H), Grade 3\&4 (I). 


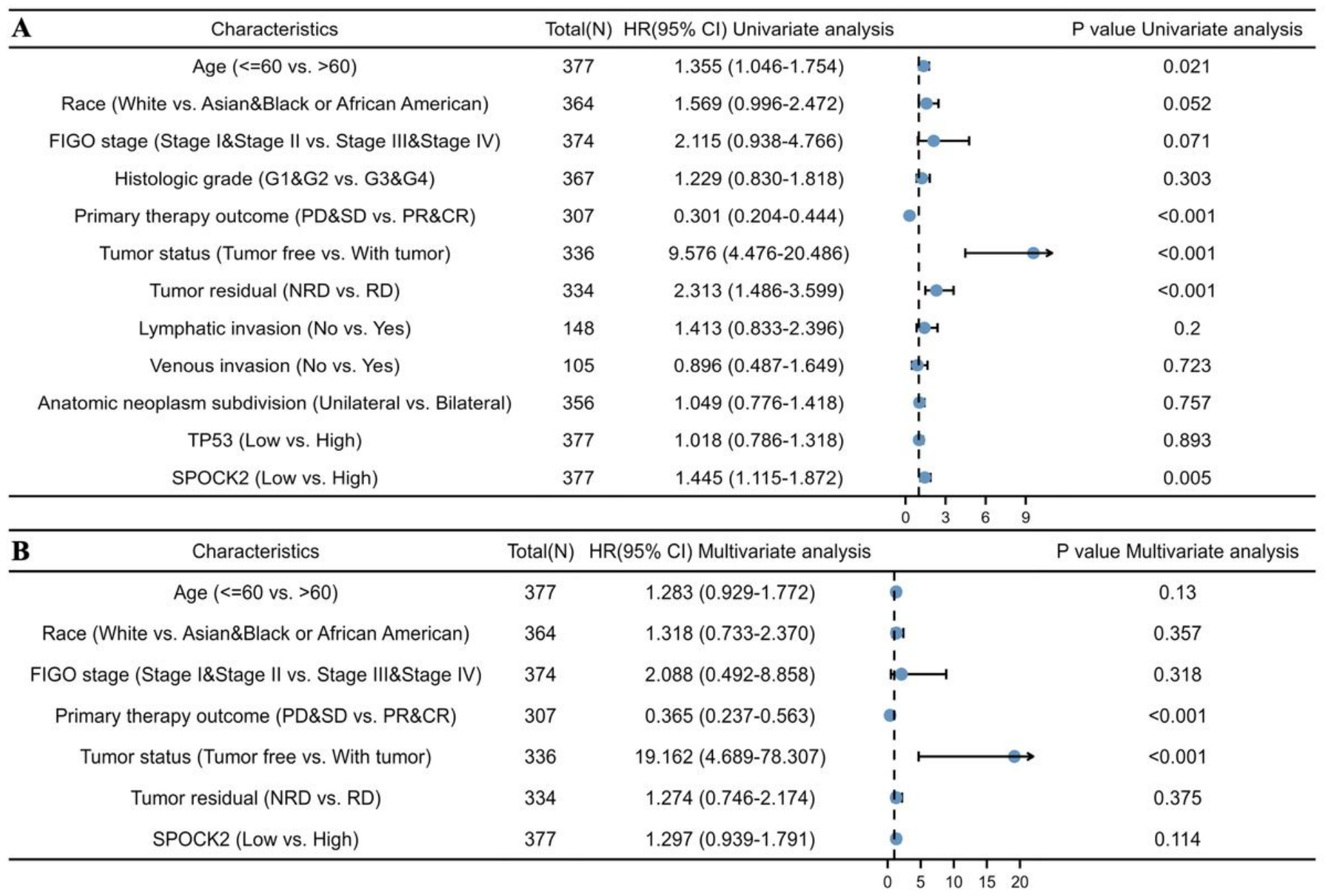

\section{Figure 5}

Univariate logistic (A) and multivariate Cox (B) regression analyses showing hazard ratios (HRs) for different clinical variables.
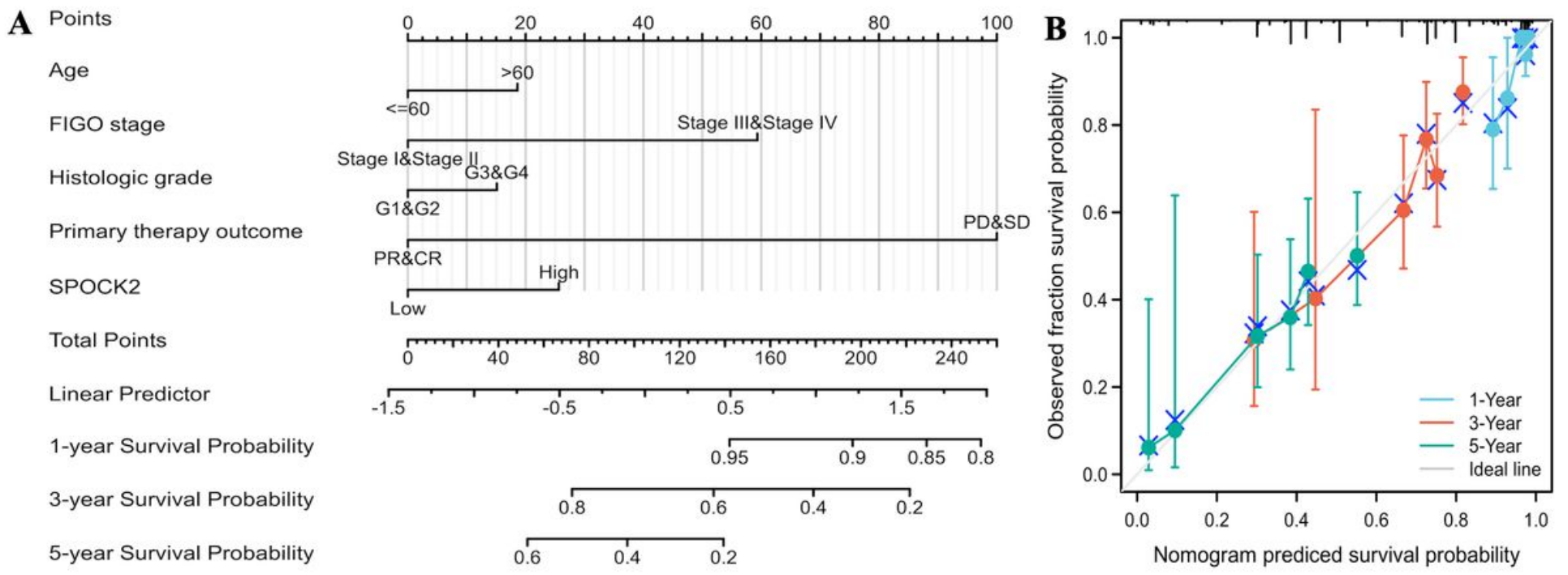

Figure 6 
Quantitative methods to predict HGSOC patients' 1-, 3-, and 5-year survival probability. A nomogram predicting 1-, 3-, and 5-year survival probability (A). Calibration plots of the nomogram for predicting the probability of OS at 1,3 , and 5 years (B).

A

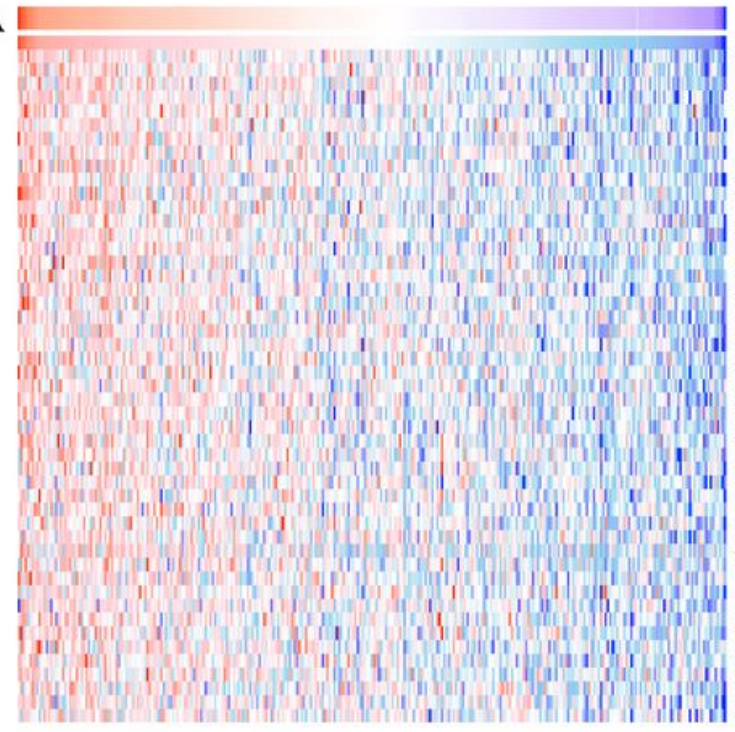

D

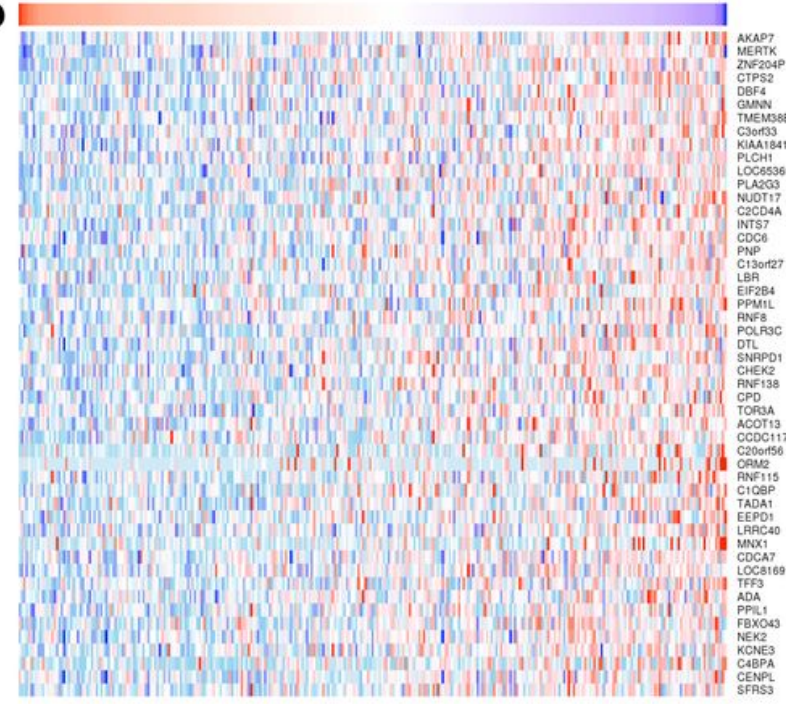

B

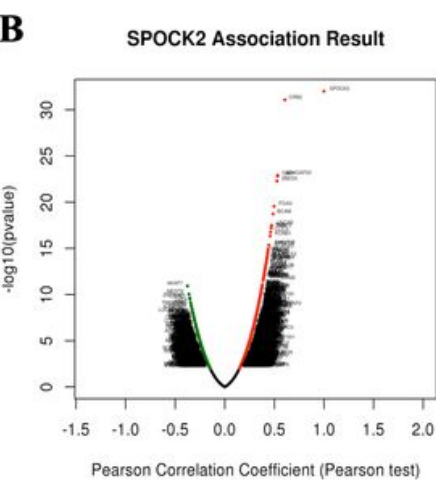

$\mathbf{E}$
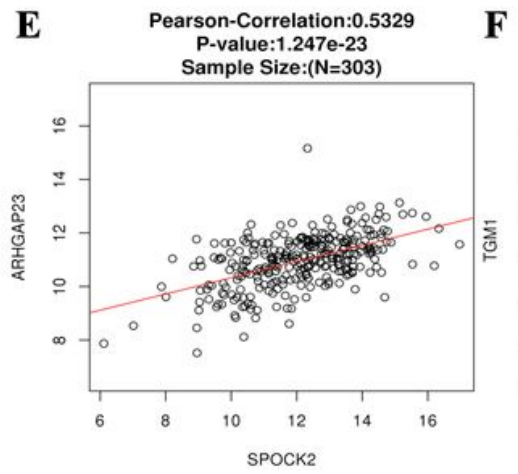

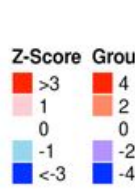

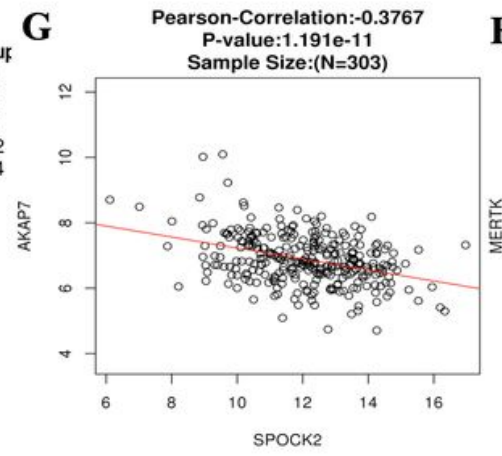

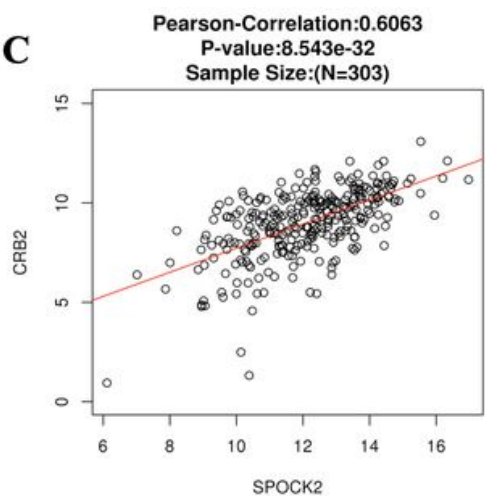

F Pearson-Correlation:0.5321 P-value:1.518e-23
Sample Size: $(\mathrm{N}=303)$

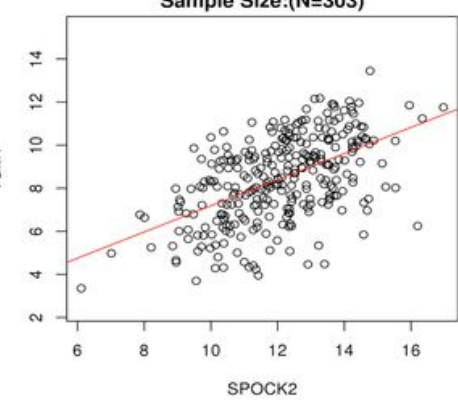

H Pearson-Correlation:-0.3612

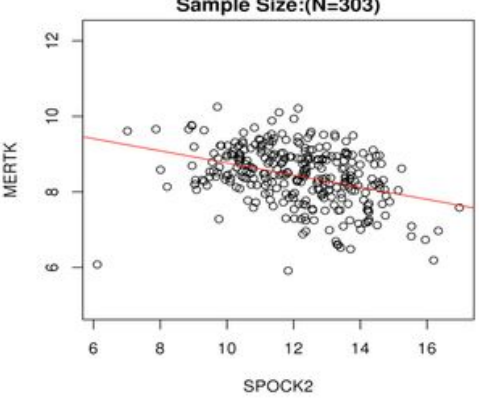

Figure 7

SPOCK2-correlated genes. All significantly correlated genes with SPOCK2 distinguished by Pearson test (B). Top 50 genes positively (A) and negatively (D) related to SPOCK2 showed by heat maps. Scatter plots presenting the most closely correlated genes of SPOCk2 with CRB2 (C), ARHGAP23 (E), TGM1 (F), $\operatorname{AKAP7}(\mathrm{G})$, and MERTK $(\mathrm{H})$. 

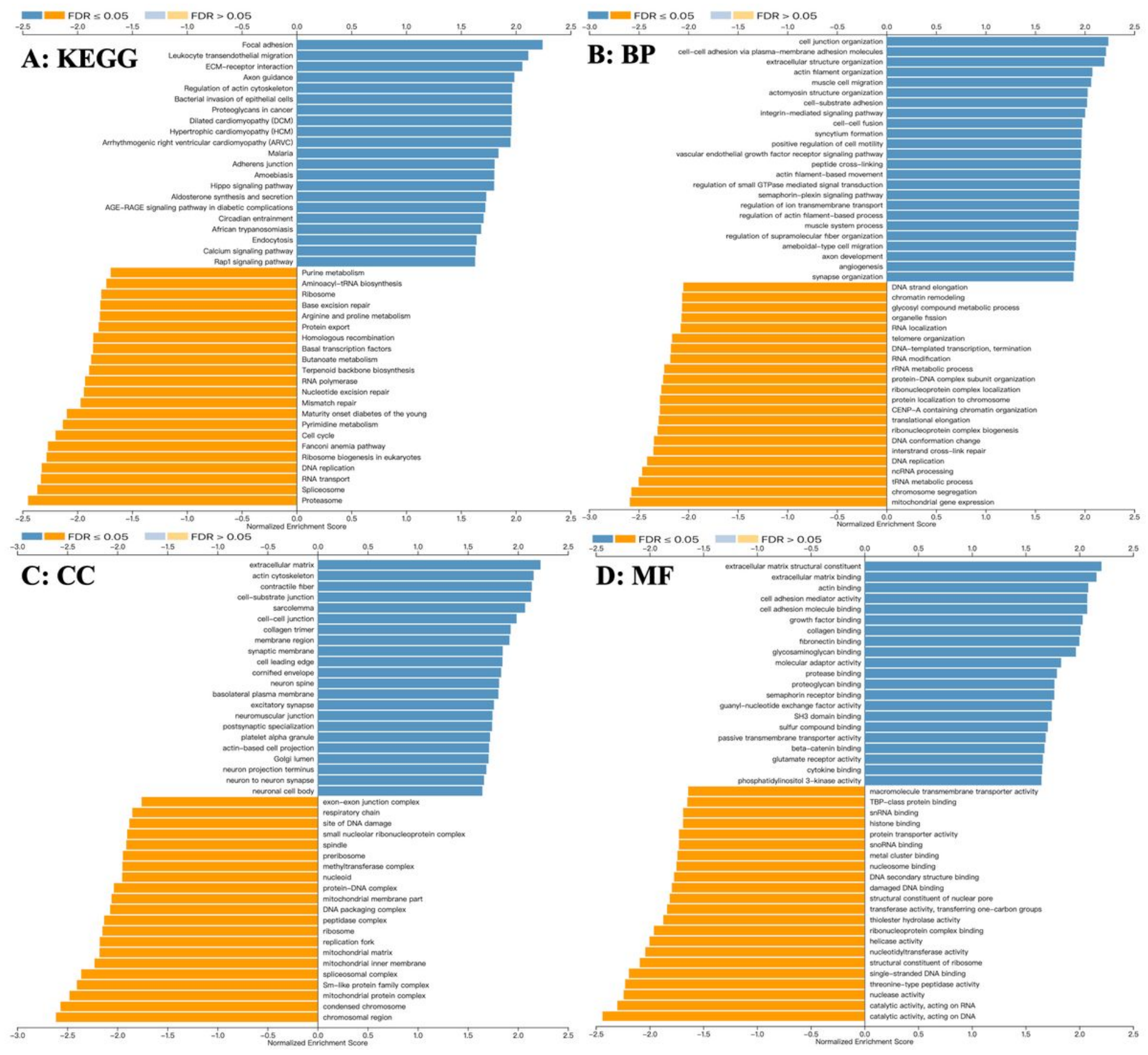

\section{Figure 8}

Functional enrichment of SPOCK2-correlated genes. Kyoto encyclopedia of genes and genomes (KEGG) $(A)$, biological process (BP) (B), cell component (CC) (C), and molecular function (MF) (D) pathway analyses. 

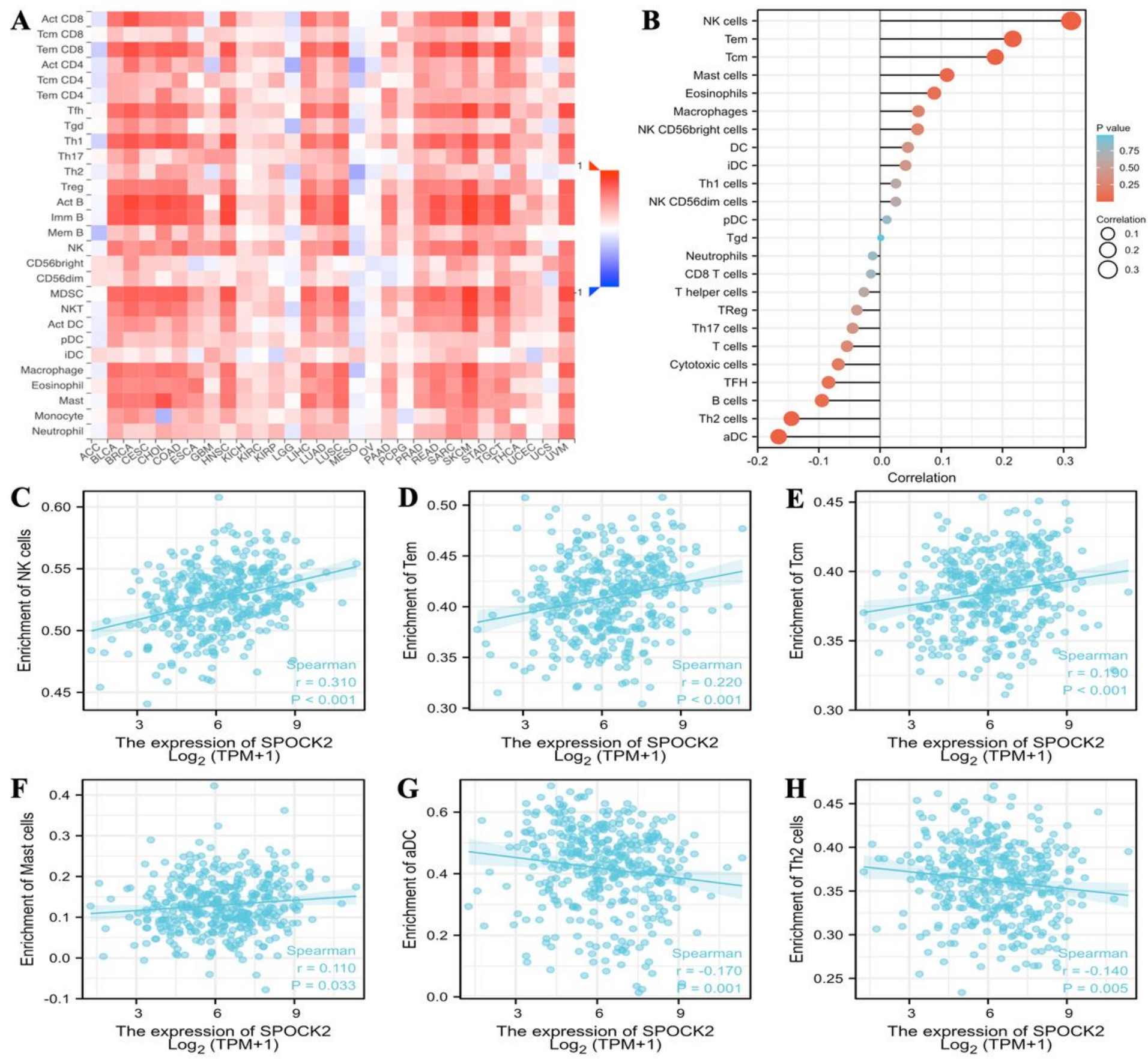

\section{Figure 9}

Correlations of SPOCK2 expression with immune infiltration level. Relations between SPOCK2 expression and tumor infiltrating lymphocytes (TILs) across human cancers (A). Correlations of SPOCK2 expression with TILs in HGSOC (B). SPOCK2 expression positively correlated with natural killer (NK) cells (C), T effector memory (Tem) cells (D), T central memory (Tcm) cells (E), and Mast cells (F); by contrast, SPOCK2 expression negatively correlated with activated dendritic cells (aDC) (G) and T helper 2 (Th2) cells $(H)$. 

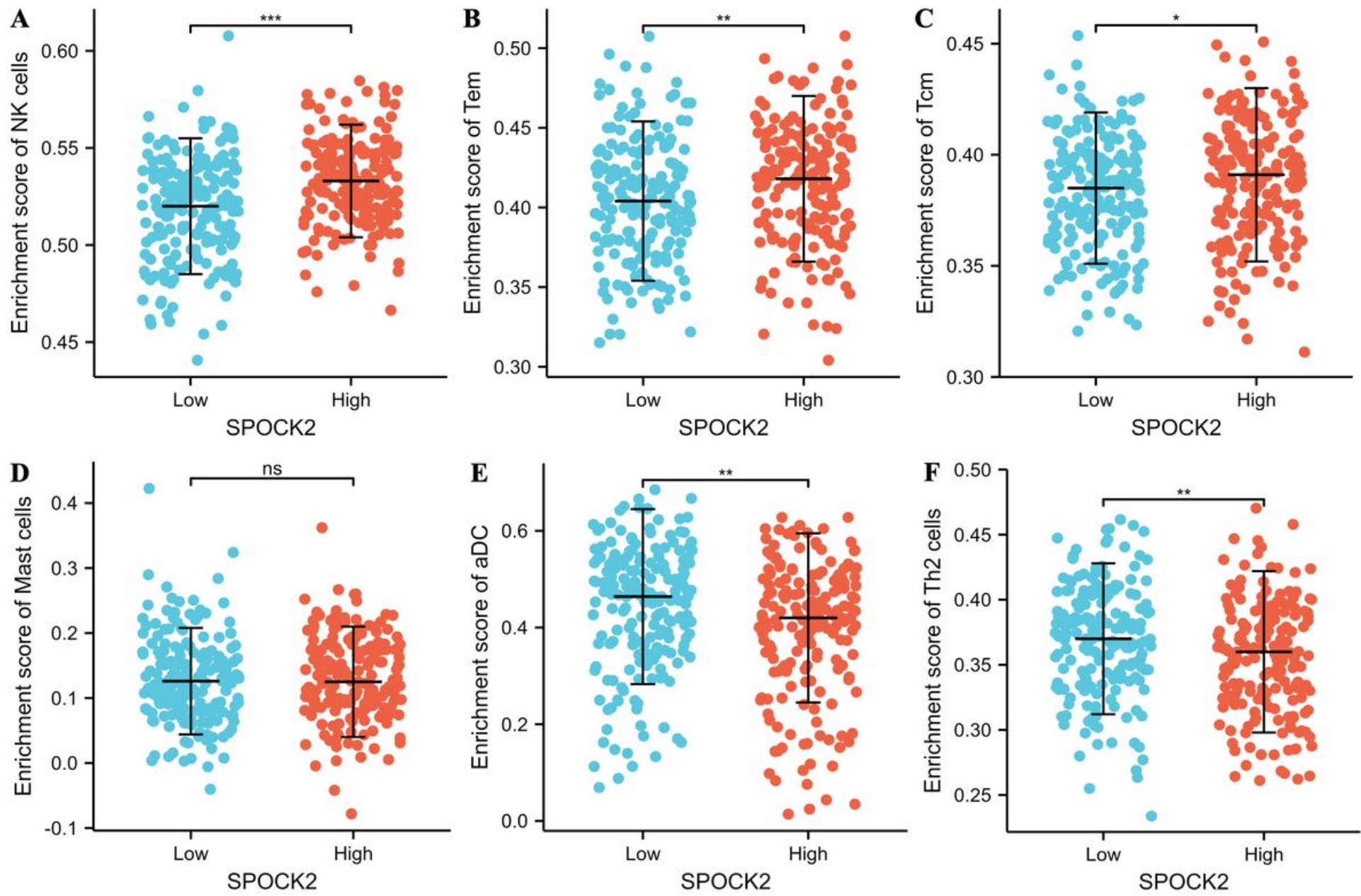

Figure 10

Abundance of NK (A), Tem (B), Tcm (C), Mast (D), aDC (E), and Th2 (F) cells between SPOCK-low and high groups.

\section{Supplementary Files}

This is a list of supplementary files associated with this preprint. Click to download.

- Table1.xlsx

- SupplementaryTable1.xlsx 